\title{
Determining the Unique Radiological Features of Lobular Breast Cancer on Imaging in Histopathologically Proven Cases - Our Institutional Experience
}

\author{
Kashifa Rahamath ${ }^{1}$, Bhawna Dev², Venkata Sai P.M. ${ }^{3}$ \\ 1, 2, 3 Department of Radiodiagnosis, Sri Ramachandra Institute of \\ Higher Education and Research, Chennai, Tamil Nadu, India.
}

\section{ABSTRACT}

\section{BACKGROUND}

Lobular breast carcinomas have always been a diagnostic challenge, over the years, to the radiologist. They are one of the most commonly missed lesions on breast cancer screening checks, due to their varied presentation. We wanted to provide a concise and practical approach to characterise their morphology and presentation on mammography and ultrasound.

\section{METHODS}

A retrospective study was done for four years and a total of 699 patients with histopathologically proven breast cancer were chosen. Those patients with invasive lobular carcinoma $(\mathrm{N}=56)$ and invasive ductal carcinoma $(\mathrm{N}=538)$ were segregated and 50 cases from each group were selected randomly.

\section{RESULTS}

On mammography, an irregular, high-density mass was the most common presentation of both lobular (68\%) and ductal (86\%) carcinomas. Presentation as focal asymmetry (28\%) was significantly more prevalent in lobular breast carcinomas. Sonographically, an architectural distortion (30\%) and non-parallel orientation (28\%) was predominantly seen in invasive lobular carcinomas (ILCs). Mass (88\%) with micro lobulated (34\%) or angular (22\%) margins was more in favour of ductal carcinoma. Other general parameters like age at presentation, positive family history, multifocality, bilaterality, tumour size and lymph nodal involvement were not significantly different between both the groups.

\section{CONCLUSIONS}

A careful analysis of digital breast tomosynthesis and ultrasonography, keeping in mind all the clear differentiating features, along with experience in the field, will dramatically increase the early detection of lobular breast cancers.

\section{KEY WORDS}

Invasive Lobular Carcinoma (ILC), Invasive Ductal Carcinoma (IDC), X-Ray

Mammography, Sono-Mammography
Corresponding Author: Dr. Bhawna Dev, A7 \& A9, Grassland Apartments, 98, Poonamalle High Road, Porur, Chennai - 600116, Tamil Nadu, India.

E-mail: bhawnadev@gmail.com

DOI: $10.14260 / \mathrm{jemds} / 2021 / 274$

How to Cite This Article:

Rahamath K, Dev B, Sai PMV. Determining the unique radiological features of lobular breast cancer on imaging in histopathologically proven cases - our institutional experience. J Evolution Med Dent Sci 2021;10(18):1296-1301, DOI: 10.14260/jemds/2021/274

Submission 29-12-2020,

Peer Review 06-03-2021,

Acceptance 12-03-2021,

Published 03-05-2021.

Copyright (c) 2021 Kashifa Rahamath et al. This is an open access article distributed under Creative Commons Attribution License [Attribution 4.0 International (CC BY 4.0)] 


\section{BACKGROUND}

Breast cancer ranks second among the most common cancers worldwide and is the most common cause of mortality due to cancers. In India, it has become the most common cancer in women due to the rapid expansion of the urban population. Due to the existing diversity in culture and lifestyles, the presentation of breast cancer is very variable in India.

The common presentation of breast abnormalities includes palpable lumps in the breast, nipple discharge, incidentally detected abnormalities on routine screening mammography or chest wall / skin changes in advanced disease. The infiltrating ductal carcinoma not otherwise specified (IDC-NOS), arising from the terminal ductal lobular unit (TDLU) is by far the most common histological subtype of breast cancer detected. Next in line, the infiltrating lobular carcinoma (ILC) accounts for approximately $5-15 \%$ of the cancers of the breast. The rest comprises of relatively less common histological variants. ${ }^{1}$ The incidence of lobular subtype of breast cancer has seen to be significantly increased in the last two decades, which can be attributed to the more prevalent use of post-menopausal hormone replacement therapy. ${ }^{2}$

The routinely employed imaging modalities for breast cancer screening / detection include a mammogram the gold standard, usually correlated with an ultrasound of the breast. This can be supplemented by contrast-enhanced digital mammography (CEDM), MR mammogram and PET-CT (Positron Emission Tomography-Computed Tomography) in cases necessitating further evaluation.

The limitations of x-ray mammogram include chances of missing a lesion in patients with a dense breast and the possibility of missing carcinomas of the lobular variety. The age of the patient also poses a limit for use of mammography. It has been suggested for use as a screening modality in patients aged above 35 - 40 years. ${ }^{3}$ From the histopathological standpoint, lobular carcinomas tend to grow in a diffuse pattern, with the tumour cells tending to invade the stroma without a strong desmoplastic reaction. These features tend to make it more difficult to be detected by physical examination as well as by mammography. The overall sensitivity of mammography in detecting lobular breast cancer ranges from around $57 \%$ to $79 \%{ }^{4}$

However, certain radiological features when picked up carefully can help in diagnosing these hidden cancers at an earlier stage.

In this article, we present the unique imaging features of lobular breast carcinoma as compared to the invasive ductal carcinomas (IDC) on digital mammography / 3D tomosynthesis and ultrasound.

\section{METHODS}

Approval for the study was obtained from the institutional research ethics committee. This was a retrospective study, for a duration of four years, between 2016 - 2020. During this period, the histopathology reports of a total of 699 cases who had undergone mastectomy and tru-cut biopsies of breast masses at our institution and diagnosed to have breast carcinoma were obtained. The results were categorized based on the histopathological type of breast cancer. Out of these total 699 cases, 538 were diagnosed with invasive ductal carcinoma (IDC), 56 of them had invasive lobular carcinoma (ILC), 49 had invasive ducto-lobular carcinoma and the rest had other types of carcinomas. From this subset, those patients who had not undergone any imaging investigation at our Institute were excluded.

The ducto-lobular variety was not included in the study to avoid overlapping features. A total of 50 cases each of invasive lobular and invasive ductal carcinoma were randomly selected. Their mammography and ultrasound images were studied and analyzed systematically to determine the distinct imaging features of lobular breast cancer and invasive ductal carcinomas.

\section{Image Acquisition}

The modalities for image acquisition included mammography Fujifilm Amulet 3D Tomosynthesis and Ultrasound-Toshiba Aplio 500. Image analysis was done retrospectively, after blinding of the histopathological diagnosis. Chi-square test or Fischer exact was performed and analysis was done using OpenEpi software.

3D tomosynthesis was performed with Fujifilm Amulet operated at an average of $30 \mathrm{kVP}$ and $15 \mathrm{mAs}$. This included the two standard views-craniocaudal and mediolateral oblique and also additional spot-compression / magnification views were taken when needed. Ultrasound of the breasts was done with a 7 - $14 \mathrm{MHz}$ transducer Toshiba APLIO 500. Image interpretation

The previously acquired images were analyzed back by two radiologists, after blinding of the histopathological diagnosis. One of the radiologists has an experience of 10 years in breast radiology and the other has 5 years of experience in the field.

\section{Statistical Analysis}

The data entry and descriptive analysis were done using Microsoft Excel (2010). The difference between various characteristics between invasive lobular and invasive ductal carcinoma was categorical and hence chi-square test or Fisher exact were performed. A P-value of $<0.05$ was considered to be statistically significant. Statistical Analysis was done using Open Epi software. ${ }^{5}$

\section{RESULTS}

Total numbers of cases diagnosed with histopathologically proven breast cancer from 2016 to 2020 were 699. Of these, $76.96 \%(\mathrm{~N}=538)$ were found to be infiltrating ductal carcinomas, $8.01 \%(\mathrm{~N}=56)$ were of the infiltrating lobular type, $7.01 \%(\mathrm{~N}=49)$ were infiltrating ducto-lobular type, 3.57 $\%(\mathrm{~N}=25)$ were ductal carcinoma in situ (DCIS), $2.14 \%(\mathrm{~N}=$ $15)$ were mucinous carcinomas and $2.28 \%(\mathrm{~N}=16)$ were invasive carcinoma with medullary features. After exclusion of those patients who had no prior imaging reports, a total of 50 patients with invasive lobular carcinoma and 50 patients with invasive ductal carcinoma were randomly chosen for the study. 
The average age of incidence was comparable in both groups with no statistically significant difference $(P=0.06)$. It was $52.3+/-9.28$ (mean + / - SD) years in the lobular cancer type and $55.7+/-8.56$ (mean $+/-$ SD) years in the ductal carcinoma type. Majority of the tumours in both groups were mostly seen in breasts with a parenchymal density B (P 0.06). Concerning other general parameters, the incidence of multifocality was more common in invasive lobular carcinomas. However, this difference was statistically insignificant $(\mathrm{P}=0.18)$. Other factors like axillary lymph nodal involvement, association with a positive family history for breast cancer and bilateral involvement were not significantly different between the two groups.

\section{Digital Mammography / 3D Tomosynthesis}

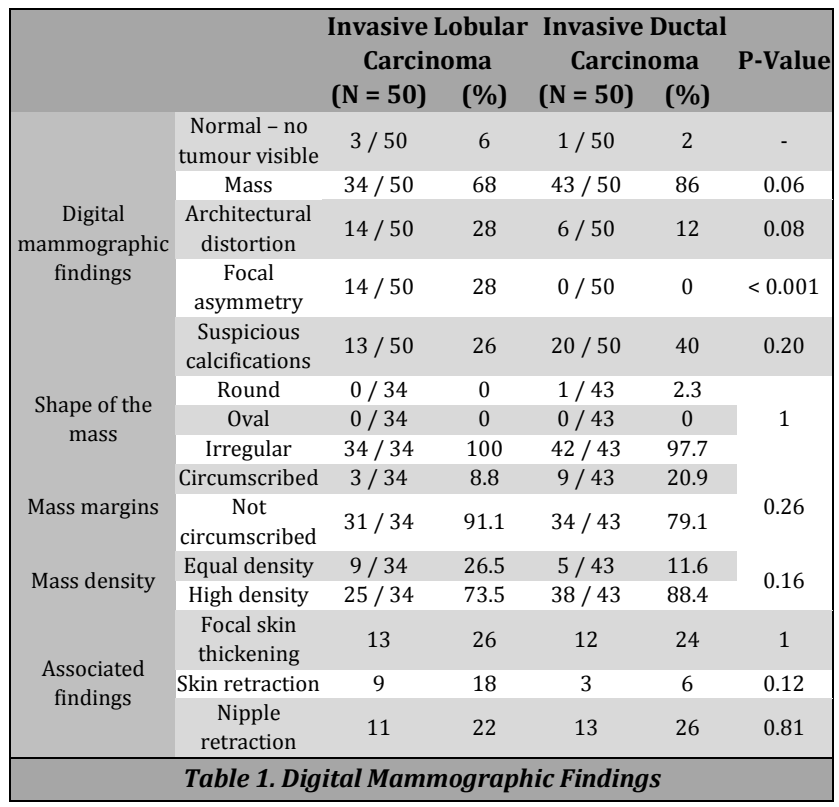

The mammographic findings of invasive lobular and ductal carcinomas are summarized in Table 1. Invasive lobular carcinomas most commonly presented as an irregular, high density mass on mammography (34 out of 50 cases - $68 \%$ ), although relatively lesser than the invasive ductal carcinoma group (43 out of 50 cases - $86 \%$ ) (Figure 3 (a, b)). This difference was found to be statistically insignificant $(\mathrm{P}=0.06)$. Characteristics of the mass like shape, margins and density were separately analysed for the 34 cases in the lobular carcinoma group and 43 cases in the ductal carcinoma group. However, no statistically significant difference could be seen. Tumour presentation as architectural distortion (Figure $1 \mathrm{~b}$, d)) (P 0.08) and focal asymmetry ( $P<0.01$ ) was associated more frequently with the invasive lobular carcinomas. However, only the increased incidence of ILCs as focal asymmetry was statistically significant. These findings were conspicuous on tomosynthesis, which played an indispensable role in their diagnosis and evaluation. 3D tomosynthesis was also helpful in excluding overlapping features which could simulate a mass. In the analysis of calcifications, those with a benign morphology-round / rim / dystrophic / rod like / popcorn like calcifications were not taken into account. Calcifications which appeared suspicious-amorphous (Figure $2(\mathrm{a}, \mathrm{b}, \mathrm{c}))$ / coarse heterogeneous / fine pleomorphic and fine linear branching were grouped under a common subheading 'suspicious calcifications'. Although the presence of suspicious calcifications was predominant in the ductal carcinomas, it was statistically insignificant $(\mathrm{P}=0.2)$. A major number of these were of fine pleomorphic and linear branching type, in a segmental or grouped distribution. No appreciable findings on mammography / tomosynthesis were seen in 3 of the ILC cases ( $6 \%$ ) and one of the IDC cases (2\%). The associated findings like focal skin thickening (Figure $2(a, b, c), 3(a, b)$ ) and nipple retraction (Figure $2(a, b, c), 3(a, b)$ ) were comparable between both the groups, except for skin retraction (Figure 1 (d)) which was mostly seen with invasive lobular carcinomas. However, this finding had no statistically significant difference $(\mathrm{P}=0.12)$.

\section{Ultrasound}

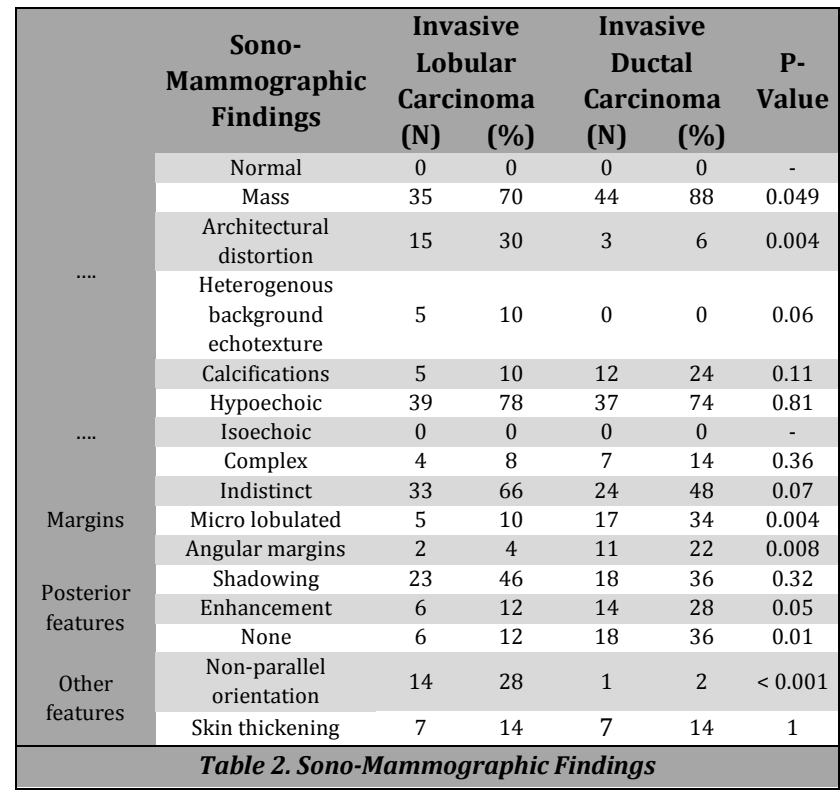

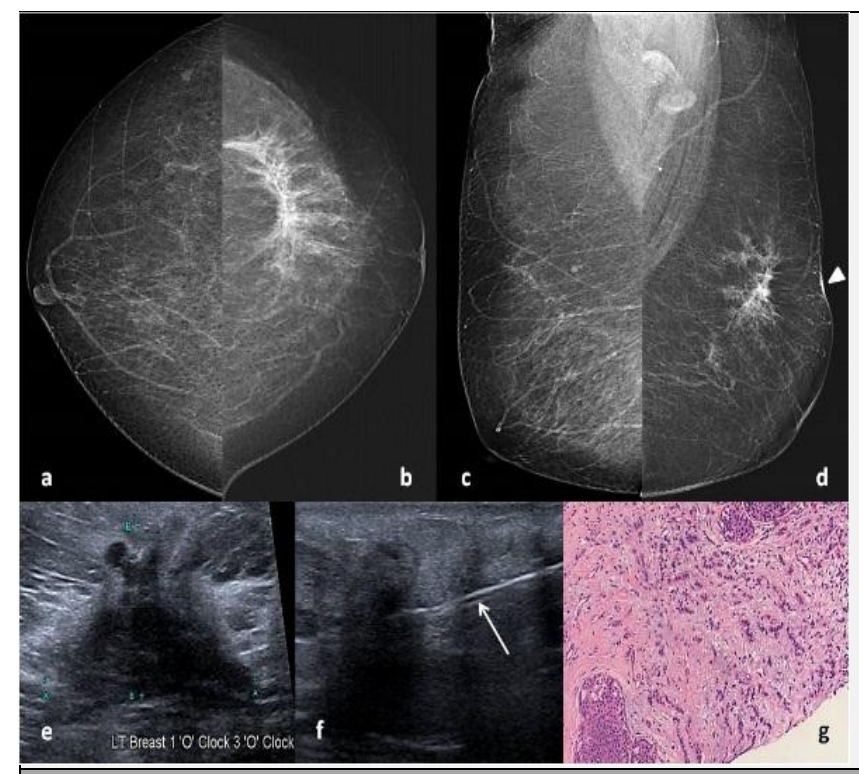

Figure 1. Mammography-CC and MLO Views of Both Breasts $(a, b, c$, d) of a 69-Year-Old Female Shows an Area of Architectural Distortion in the Upper Outer Quadrant of the Left Breast, with Associated Skin Retraction (arrowhead). Ultrasound sonograph Shows an Ill-Defined Area of Vague Abnormality (e), with Posterior Acoustic Shadowing. USG Guided Biopsy Performed (f) (arrow) and Histopathology Proved It to Be Invasive Lobular Carcinoma (g). 
The findings on sonomammography are summarized in Table 2. One of the cases of invasive ductal carcinoma group presented with a complex cystic lesion and was excluded from the study to avoid a statistical error. On ultrasound, both the groups were commonly seen as an indistinct, hypoechoic mass, but the incidence was higher in the ductal carcinoma group. This difference was found to be statistically significant $(P=0.049)$ (Figure 2 (d), 3 (c, d)).

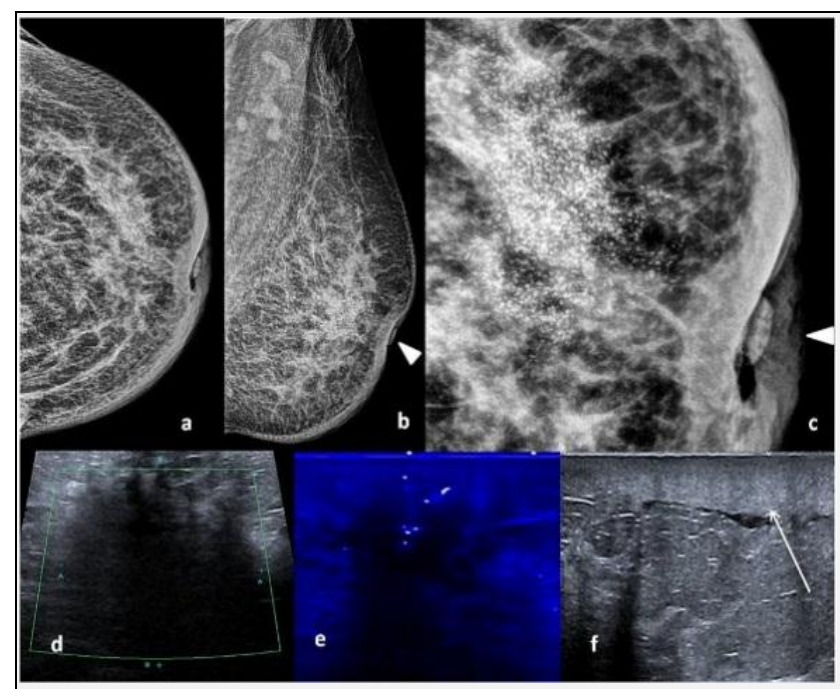

Figure 2. Digital Mammography-CC and MLO Views of Left Breast $(a, b)$ of a 60-Year-Old Female Show Grouped, Amorphous Calcifications (Zoomed in Image-c) in the Upper Central Quadrant of Left Breast, Extending to the Nipple. Skin Thickening Seen Involving the Lower Inner Quadrant, with Nipple Retraction (Arrowheads). USG Shows an Irregular, Ill-Defined Hypoechoic Mass in the Subareolar Region (d), with Few Microcalcifications (e). Skin and Subcutaneous Thickening Seen (f) (arrow). The Patient was Diagnosed with Invasive Lobular Carcinoma.

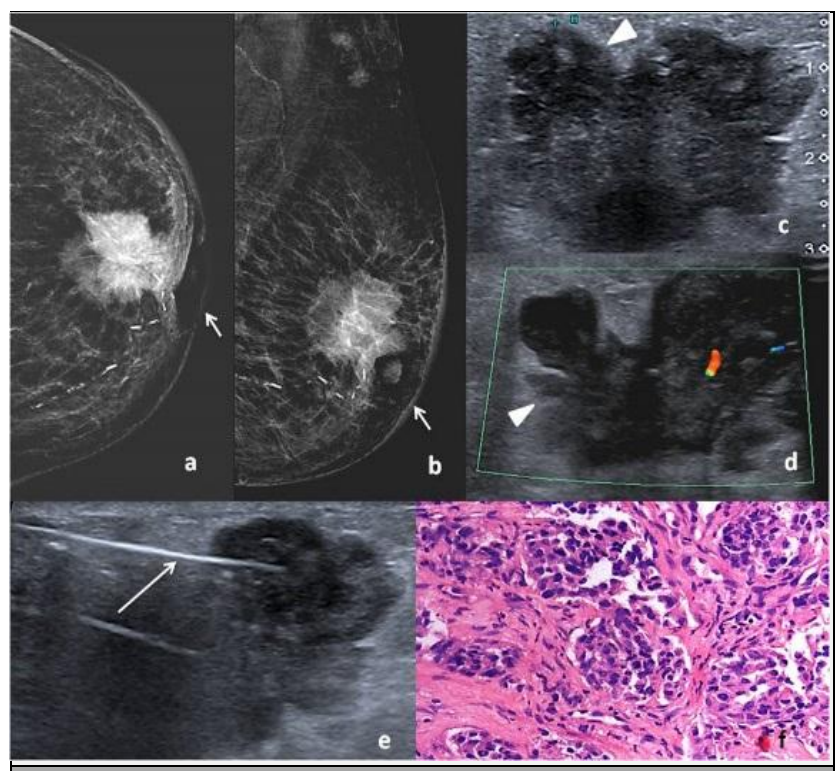

Figure 3. Digital Mammography CC and MLO Views of the Left Breast $(a, b)$ of a 64-Year-Old Female Showing an Irregular, HighDensity, Speculated Mass in the Central Quadrant. Skin Thickening and Nipple Retraction Seen in the Peri Areolar Region (Short Arrows). US Correlation Shows an Irregular Shaped Hypoechoic Lesion (c, d), with Angular Margins (Arrowheads) and Internal Vascularity (d). USG Guided Biopsy of the Lesion (e) (Long Arrow) was Reported as Invasive Ductal Carcinoma of the Breast ( $f$ )
Few of the lobular carcinomas were also just seen as an architectural distortion (30\%) (P=0.04) (Figure $1(\mathrm{e})$ ) or with a heterogeneous background echotexture $(10 \%)(\mathrm{P}=0.06)$. The presentation as architectural distortion was seen to have a statistically significant difference. Calcifications were predominantly seen in the ductal carcinoma group, akin to digital mammography.

The masses of invasive ductal carcinoma were seen to have micro lobulated (P 0.004) or angular margins 3 (c, d)) ( 0.008). While a slightly higher percentage of the ductal carcinomas were associated with posterior acoustic enhancement (28\%) ( $\mathrm{P}=0.05)$, a significant number of them did not have any posterior features (36\%) as compared to the lobular cancers $(\mathrm{P}=0.01)$. The masses of the lobular carcinoma category had a non-parallel orientation $(\mathrm{P}<0.001)$. Associated findings like skin thickening (Figure 2 (f)) were almost equal between both the groups (P 1).

\section{DISCUSSION}

The invasive lobular carcinomas account for the secondlargest subset among the invasive breast cancers, first being the ductal variety. These tumours are mostly detected at a later stage, when the tumour is larger. The radiological diagnosis and management of lobular breast cancers is quite challenging. Clinically, lobular cancers are mostly not palpable, as they do not form discrete masses. These tumours arise from the peripheral lobular epithelial cells, leading to secondary cancerization of ducts and lobules. They are insidious in onset and do not evoke a strong desmoplastic reaction.

Pathologically, the tumour cells are small and have a round nucleus with scanty cytoplasm. Immunohistochemically, loss of E-Cadherin adhesion molecule is seen, which is one of the most supporting factors leading to diagnosis.6,7 Low Ki67 is seen, which indicates a lower proliferation rate. This, in turn, favours a slightly better prognosis and a longer disease-free survival rate.

Lobular breast cancers have a propensity to spread to the adrenals. Other sites of metastases include the leptomeninges, gastrointestinal tract, retroperitoneum and myocardium. Lung metastasis is lesser as compared to the invasive ductal carcinomas.

The low sensitivity of mammography and ultrasound in diagnosing cancers of the lobular variety, has been reported by many. Berg WA et al. ${ }^{8}$ have stated a mammographic sensitivity of $81 \%$ in detecting invasive ductal carcinomas and only $34 \%$ in invasive lobular carcinomas. This has led to the increased usage of higher imaging modalities like magnetic resonance imaging (MRI) for evaluation of patients with an increased risk of breast cancer, especially the lobular variety.

Our study was designed to recognize the characteristic imaging features of lobular breast carcinoma in comparison with the ductal carcinomas, on mammography and ultrasound, which would enhance their detection and survival rate.

Out of a total of 699 patients with histopathologically proven breast cancer, 594 patients had either invasive ductal $(\mathrm{N}=538)$ or invasive lobular $(\mathrm{N}=56)$ carcinomas. Rest of the 105 patients had mixed ducto-lobular carcinoma $(\mathrm{N}=49)$, in situ ductal carcinoma ( $\mathrm{N}=25)$, mucinous cancers $(\mathrm{N}=15)$ and other less common subtypes. Patients who did not undergo any imaging investigations in our department or underwent 
imaging with only one modality were excluded from the study. Of the total 594 patients, who had either invasive ductal or invasive lobular carcinomas, 50 patients from each group were randomly selected and taken up for the study.

Before the interpretation of the images, it was made sure that the images were taken properly and satisfactory coverage of all parts was ensured. No artefacts were entertained.

The first step of interpretation was to look at the breast density and grade it according to ACR BI-RADS. Involvement of dense breasts background parenchymal density category $C$ and D was seen to be common in lobular carcinomas, although the difference was not statistically significant.

Multifocality and bilateral incidence have been reported more frequently in the invasive lobular breast cancers, ${ }^{9}$ which was also observed in our study. A preponderance in the older age group is common, which can be attributed to the low detection rates and the effect of post-menopausal status. ${ }^{10}$ However, the mean age group of incidences was comparable between the lobular and ductal carcinomas in our study.

In accordance with other studies, ${ }^{11,12}$ on mammography, both the ductal (86 \%) and lobular (68\%) carcinomas were commonly identified as an irregular, equal or high-density mass, with non-circumscribed margins. Kim SH et al.12 conducted a comparative study between 27 patients with invasive lobular carcinoma and 85 patients with invasive ductal carcinoma. The most common mammographic presentation of both these types of tumours was that of an irregular, isodense mass with non-circumscribed borders (59 $\%$ of ILCs and $45 \%$ of IDCs). Cornford EJ et al. ${ }^{11}$ compared 86 cases each of ILCs and IDCs and found that a spiculated mass was the most common feature in both the groups $(69 \%$ and $63 \%$ respectively). No specific difference in shape, margins or density of the mass was found between both categories in our study.

Microcalcifications as an aid to diagnosis in various formspleomorphic / fine linear / branching patterns, were present in only $26 \%$ of our patients with invasive lobular carcinoma, as compared to $40 \%$ in ductal carcinomas in our study, as also seen in other studies. This is in favour of the conception that ILCs generally have less frequent calcifications. In an analysis of 94 biopsy proven invasive lobular carcinoma lesions by Evans WP et al., ${ }^{13}$ only $20 \%$ of them were described as asymmetric densities or calcifications. Lopez JK and Bassett $L W,{ }^{9}$ in a retrospective review of 49 patients with invasive lobular cancer, found calcifications to be associated with $16 \%$ of the cases. This can be attributed to being one of the causes for lower detection rates of lobular cancers on mammography. 14

The other common presentations of invasive lobular carcinomas in our current study include architectural distortion (28\%) and focal asymmetry / asymmetric densities (28\%), as supported by the literature.13,15 This was significantly higher as compared to the ductal carcinoma group, where architectural distortion was seen in $12 \%$ and focal asymmetry was not identified in any.

False-negative results or no appreciable findings on mammography have been reported to be between $14-19$ $\%$ in lobular breast carcinomas.7,8 However, in our study, no detectable abnormality could be seen in $6 \%$ of ILCs.

With regards to the associated secondary mammographic findings like skin thickening and skin / nipple retraction, not much difference has been observed between the lobular and ductal cancers in our study, which holds good in earlier studies as well. ${ }^{11}$

The most commonly reported US findings of lobular carcinomas, as stated in literature include an indistinct, hypoechoic mass with posterior acoustic shadowing. ${ }^{9,12,15} \mathrm{Kim}$ $\mathrm{S} \mathrm{H}$ et al. ${ }^{12}$ in their comparative study between invasive lobular and ductal carcinomas, proved that both the cancers were seen as an irregular hypoechoic mass with spiculated margins, except that posterior acoustic shadowing was mostly associated with lobular cancers. Similar features were identified in our study. However, presentation as a mass was more frequently seen with ductal carcinomas than lobular carcinomas, which is also seen to exhibit a statistically significant difference in our analysis.

In a study by Selinko et al. ${ }^{16} 13 \%$ of the ILCs have been identified as an ill-defined area of inhomogeneous and altered echotexture. No distinct margins could be identified. Similarly, $30 \%$ of the ILCs in our study were appreciated as an architectural distortion on ultrasound imaging. The other interesting and distinguishing feature observed in our study was the presence of micro lobulated (34\%) and angular margins (22\%) to be more prevalent in IDCs. These findings were identical to a study done by Rotstein AH and Neerhut PK in 2005. ${ }^{17}$

Although the classic malignant feature of posterior acoustic shadowing is common with ductal cancers, in our study, an almost equal number of patients with IDC were seen with no distinct posterior features (36\%). This was in contrast to that of the lobular cancers, where only $6 \%$ of the tumours did not show any distinct posterior features. Whilst being uncommon, concordant findings with ductal carcinomas have been recorded by Rotstein $\mathrm{AH}$ et al. ${ }^{17}$ in a retrospective analysis of 181 cases with IDC, where $48 \%$ of the tumours had neither posterior shadowing nor enhancement.

A non-parallel orientation of the lesion on ultrasound has been commonly described to be associated with invasive ductal carcinomas. ${ }^{18}$ Lobular carcinomas are mostly seen in a parallel orientation, due to the growth pattern of the tumor. ${ }^{19}$ On the contrary, in our study, a non-parallel orientation of the tumour was frequently identified in the ILC group. Jones KN et al., ${ }^{20}$ in their study on pure ILCs presenting as a hyperechoic mass, have described non-circumscribed margins and nonparallel orientation to be reliable predictors of malignancy, similar to our study.

We found that ultrasound has an upper hand over mammography in differentiating ductal and lobular cancers. With regards to the limitations of the current study, the smaller sample size is the only drawback. Further prospective studies with a larger sample volume would help overcome this limitation.

Thus, a careful analysis of the morphology of the lesions on mammography and ultrasound, keeping in mind the above discussed features would help us derive a conclusion that a particular cancer could be of the lobular variety.

\section{CONCLUSIONS}

Comparing the imaging features of the invasive ductal breast carcinomas, the commonest histological variety \& invasive lobular carcinoma, a few unique imaging features were found 
useful to better characterize the lobular carcinomas. These include:

- A focal asymmetry detected on mammography may indicate invasive lobular carcinoma.

- Architectural distortion identified on sonography favours lobular carcinomas.

- Non-parallel orientation of a lesion on ultrasound is more commonly associated with ILC.

- Presentation as mass, especially with micro lobulated / angular margins on ultrasonography is less prevalent in ILC.

However, histopathology is the gold standard in confirming the diagnosis.

Data sharing statement provided by the authors is available with the full text of this article at jemds.com.

Financial or other competing interests: None.

Disclosure forms provided by the authors are available with the full text of this article at jemds.com.

\section{REFERENCES}

[1] Nikumbh DB, Kanthikar SN, Suryawanshi KH, et al. Histopathological spectrum of unusual breast lesions: a seven year retrospective review. Indian Journal of Pathology and Oncology 2016;3(3):456-62.

[2] Rosenberg LU, Magnusson C, Lindström E, et al. Menopausal hormone therapy and other breast cancer risk factors in relation to the risk of different histological subtypes of breast cancer: a case-control study. Breast Cancer Res 2006;8(1):R11.

[3] Dixon JM, Mansel RE. Symptoms, assessment and guidelines for referral. In: Dixon JM, ed. $\mathrm{ABC}$ of breast diseases. $2^{\text {nd }}$ edn. London: BMJ Books 2000: p. 3-7.

[4] Porter AJ, Evans EB, Foxcroft LM, et al. Mammographic and ultrasound features of invasive lobular carcinoma of the breast. J Med Imaging Radiat Oncol 2014;58(1):1-10.

[5] Dean AG, Sullivan KM, Soe MM. OpenEpi: Open Source Epidemiologic Statistics for Public Health, Version 3.01. www.OpenEpi.com updated 06-04-2013, acessed on 1803-2021.

[6] Dabbs DJ, Schnitt SJ, Geyer FC, et al. Lobular neoplasia of the breast revisited with emphasis on the role of Ecadherin immunohistochemistry. Am J Surg Pathol 2013;37(7):e1-11.

[7] Lee JH, Park S, Park HS, et al. Clinicopathological features of infiltrating lobular carcinomas comparing with infiltrating ductal carcinomas: a case control study. World J Surg Oncol 2010;8:34.

[8] Berg WA, Gutierrez L, NessAiver MS, et al. Diagnostic accuracy of mammography, clinical examination, US and MR imaging in preoperative assessment of breast cancer. Radiology 2004;233(3):830-49.

[9] Lopez JK, Bassett LW. Invasive lobular carcinoma of the breast: spectrum of mammographic, US and MR imaging findings. Radiographics 2009;29(1):165-76.

[10] Li CI, Anderson BO, Porter P, et al. Changing incidence rate of invasive lobular breast carcinoma among older women. Cancer 2000;88(11):2561-9.

[11] Cornford EJ, Wilson AR, Athanassiou E, et al. Mammographic features of invasive lobular and invasive ductal carcinoma of the breast: a comparative analysis. $\mathrm{Br}$ J Radiol 1995;68(809):450-3.

[12] Kim SH, Cha ES, Park CS, et al. Imaging features of invasive lobular carcinoma: comparison with invasive ductal carcinoma. Jpn J Radiol 2011;29(7):475-82.

[13] Evans WP, Burhenne LJW, Laurie L, et al. Invasive lobular carcinoma of the breast: mammographic characteristics and computer-aided detection. Radiology 2002;225(1):182-9.

[14] Kopans DB. Malignant lesions of the lobule. In: Kopans DB, ed. Breast imaging. Baltimore, MD: Lippincott Williams \& Wilkins 2007:866-70.

[15] Johnson K, Sarma D, Hwang ES. Lobular breast cancer series: imaging. Breast Cancer Res 2015;17(1):94.

[16] Selinko VL, Middleton LP, Dempsey PJ. Role of sonography in diagnosing and staging invasive lobular carcinoma. J Clin Ultrasound 2004;32(7):323-32.

[17] Rotstein AH, Neerhut PK. Ultrasound characteristics of histologically proven grade 3 invasive ductal breast carcinoma. Australas Radiol 2005;49(6):476-9.

[18] Blaichman J, Marcus JC, Alsaadi T, et al. Sonographic appearance of invasive ductal carcinoma of the breast according to histologic grade. AJR Am J Roentgenol 2012;199(3):W402-8.

[19] Kombar OR, Fahmy DM, Brown MV, et al. Sonomammographic characteristics of invasive lobular carcinoma. Breast Cancer (Dove Med Press) 2012;4:11524.

[20] Jones KN, Magut M, Henrichsen TL, et al. Pure lobular carcinoma of the breast presenting as a hyperechoic mass: incidence and imaging characteristics. AJR Am J Roentgenol 2013;201(5):W765-9. 